\title{
20
}

\section{An M/M/c Queue with Interdependent Arrival and Service Processes}

\author{
K. Yoneyama ${ }^{*}$, T. Miyamura ${ }^{* *}$ and H. Ishii ${ }^{* * *}$ \\ ${ }^{*}$ Department of Management and Informatics, Himeji College of Hyogo, \\ 1-1-12 Shinzaike-Hon-Cho, Himeji, Hyogo 670-0092 Japan, \\ Phone:+81-792-92-1515, Fax:+81-792-93-5710, \\ E-mail:yoneyama@hjc.himej̈-tech.ac.jp \\ ** Faculty of Engineering, Osaka University, \\ 2-1 Yamada-Oka, Suita, Osaka 565-0871 Japan, \\ Phone:+81-6-877-5111(ext.3641), Fax:+81-6-879-7871, \\ E-mail:miyamura@mild.ap.eng.osaka-u.ac.jp \\ ${ }^{* * *}$ Faculty of Engineering, Osaka University, \\ 2-1 Yamada-Oka, Suita, Osaka 565-0871 Japan, \\ Phone:+81-6-879-7868, Fax:+81-6-879-7871, \\ E-mail:ishiiha@ap.eng.osaka-u.ac.jp
}

\begin{abstract}
Most practical queueing systems have dependencies between arrival and service patterns. In this paper we consider the $M / M / c$ queueing system with interarrival and service times having the multivariate exponential distribution of Marshall and Olkin. We derive the standard representation of the multivariate exponential distribution of Marshall and Olkin, and calculate the steady state probability vector by a matrix-geometric approach, and study a monotonic tendency of dependency effect on some random variable.

\section{Keywords}

Multivariate exponential distribution, multivariate phase type distribution, standard representation, $\mathrm{M} / \mathrm{M} / \mathrm{c}$ queueing system, matrix-geometric approach
\end{abstract}

Performance and Management of Complex Communication Networks

T. Hasegawa, H. Takagi \& Y. Takahashi (Eds.) @ 1998 IFIP. Published by Chapman \& Hall 


\section{INTRODUCTION}

In this paper we consider a multiserver queueing system with an interarrival time and $c$ service times having exponential distributions. However, they are not independent. The service times of $c$ channels are correlated to an interarrival time. Any channel does not only finish service independently on an arrival as the ordinary $\mathrm{M} / \mathrm{M} / \mathrm{c}$ queueing system, but also all $c$ channels sometimes finish service simultaneously when a customer arrives. It is encountered in practice because when the service tasks of those customers who joined the service facility are long, they are accomplished simultaneously due to the competition or cooperation among servers on an arrival, it creates dependency between an interarrival time and those service times. Given that the marginal distributions of interarrival and service times are fixed, we are concerned with how the dependency affects some random variable.

Most of queueing literature assumes independency between arrival and service patterns, though it is hardly justifiable in realistic phenomena. There exist some exceptions such as Conolly and Choo (1979), Hadidi (1981) and Langaris (1987). In those papers the authors consider a single server queue and assume that the interarrival and service times take the class of WicksellKibble bivariate exponential distribution which is defined using Bessel functions. The Laplace transform of the waiting time distribution is obtained in terms of the solution of some recursive equations, hence it is hard to derive the effect of the dependency. On the contrary, Chao (1995) assumed that the interarrival and service times have the class of bivariate exponential distributions defined by Marshall and Olkin (1967). He defined dependency between the interarrival and service times as the correlation coefficient between them. He showed that the customer waiting time is monotonically decreasing in the dependency in increasing convex ordering. Yoneyama et al.(1997) considered a two-server queueing system with an interarrival time and two service times having the class of multivariate exponential distributions of Marshall and Olkin (1967). They defined dependency between an interarrival time and two service times as the rate of the simultaneous occurrence of an arrival and service completion of two channels. They calculated the steady state probability vector by a matrix-geometric approach and studied a monotonic tendency of dependency effect on some random variable.

So far as we know, Nishida et al.(1974) first discussed the application of the bivariate exponential (abbreviated as BVE) distribution of Marshall and Olkin (1967) in the queueing theory. They considered a two-server queueing system in which their service distribution is the BVE and obtained the steady state probabilities by a generating function approach. As an extension of their model, Nishida and Yoneyama (1977) considered a multiserver queueing system whose service distribution is the multivariate exponential (abbreviated as MVE) distribution of Marshall and Olkin (1967) and derived how to get the steady state probabilities by a generating function approach. For this model, Yoneyama (1996) obtained both the queueing and waiting time distribution 
and showed that the so-called Little's law holds for their mean. Moreover, for the same model, Yoneyama et al.(1997) calculated the rate matrix R explicitly by a matrix-geometric approach and showed that the steady state probabilities whose state takes as the number of customers in the system using the rate matrix $R$ coincide with the above probabilities obtained by Nishida and Yoneyama (1977).

Assaf et al.(1984) showed that the BVE is a bivariate phase type (abbreviated as $\mathrm{BPH}$ ) distribution and its multivariate extension is a multivariate phase type (abbreviated as MPH). Raftery (1984) introduced a continuous multivariate exponential distribution and O'Cinneide and Raftery (1989) showed that it is MPH and derived its MPH representation.

In this paper, as an extension of the model introduced by Yoneyama et al. (1997), we consider a multiserver queueing system with an interarrival time and $c$ service times having the class of multivariate exponential distributions of Marshall and Olkin (1967). We define dependency between an interarrival time and $c$ service times as the rate of the simultaneous occurrence of an arrival and service completion of all channels. For this system we calculate the steady state probability vector by a matrix-geometric approach and show that the steady state probabilities for a three-server queueing system whose state takes as the number of customers in the system coincide with those of the ordinary $M / M / 3$ queueing system when an interarrival time and three service times have no dependency. Furthermore, we show that the subtraction of an interarrival time from the minimum of $c$ service times is monotonically decreasing in the dependency in increasing convex ordering. Before we describe the model fully, we derive the standard MPH representation of the MVE and get the BVE for the simplest case.

\section{STANDARD MPH REPRESENTATION OF MVE}

We will say that nonnegative random variables $S_{1}, \cdots, S_{n}$ have a joint distribution with exponential minimums if

$P\left\{\min _{i \in I} S_{i}>s\right\}=e^{-\theta_{I} s}, \quad s \geq 0$,

for some $\theta_{I}>0$ and for all nonempty sets $I \subset\{1, \cdots, n\}$. The most important example of a distribution with exponential minimums is the multivariate exponential (MVE) distribution of Marshall and Olkin (1967). Random variables $U_{1}, \cdots, U_{n}$ having this distribution can be generated by letting

$U_{i}=\min _{j \in J_{i}} X_{j}, \quad i=1, \cdots, n$,

where $J_{i} \subset\{1, \cdots, k\}$ and the random variables $X_{1}, \cdots, X_{k}$ are independent and exponentially distributed.

We will say that the joint distribution of $S_{1}, \cdots, S_{n}$ and of $U_{1}, \cdots, U_{n}$ are marginally equivalent in minimums if 


$$
P\left\{\min _{i \in I} S_{i}>s\right\}=P\left\{\min _{i \in I} U_{i}>s\right\}, \quad s \geq 0,
$$

for each nonempty set $I \subset\{1, \cdots, n\}$. Esary and Marshall (1974) showed that if $S_{1}, \cdots, S_{n}$ are random variables whose joint distribution has exponential minimums, then there exist random variables $U_{1}, \cdots, U_{n}$ with a multivariate exponential distribution such that the joint distribution of $S_{1}, \cdots, S_{n}$ and of $U_{1}, \cdots, U_{n}$ are marginally equivalent in minimums.

Assaf et al.(1984) first formulated a multivariate phase type (MPH) distribution in the following way. Suppose $\{V(t): t \geq 0\}$ is a regular Markov chain with finite state-space $E$. Let $\Gamma_{1}, \cdots, \Gamma_{n}$ be $n$ non-empty subsets of $E$ such that once $V$ enters $\Gamma_{i}$ it never leaves. Suppose that $\bigcap_{i=1}^{n} \Gamma_{i}$ consists of one state $\Delta$, into which absorption is certain. Let $\beta$ be an initial probability vector on $E$, which puts all its mass on states in $E \backslash\{\Delta\}$.

The infinitesimal generator $Q$ of $V$ is of the form

$$
\mathbf{Q}=\left[\begin{array}{cc}
\mathbf{T} & -\mathbf{T e} \\
\mathbf{0} & \mathbf{0}
\end{array}\right]
$$

where $\mathbf{T}$ is a square matrix, $\mathbf{e}$ is a column vector of ones, and $\mathbf{0}$ is a row vector of zeros. Define $Y_{i}=\inf \left\{t: V(t) \in \Gamma_{i}\right\}(i=1, \cdots, n)$. Then the distribution of $\left(Y_{1}, \cdots, Y_{n}\right)$ is MPH.

Assaf et al.(1984) showed that since $X_{i}$ is $\mathrm{PH}, U_{i}$ is $\mathrm{PH}$ and, hence, the MVE is MPH. It is of interest to give the standard MPH representation of the MVE, using the results of Assaf et al.(1984).

To derive the standard MPH representation of the MVE, we specify explicitly the ingredients $E, \Gamma_{1}, \cdots, \Gamma_{n}, \mathbf{T}$ and $\beta$. The state-space is $E=$ $\{1, \cdots, m, \Delta\}$, with $2^{n}$ elements $\mathbf{q}=\left(q_{1}, \cdots, q_{n}\right)$, where $q_{i} \in\{0,1\}$ and $m=2^{n}-1$. For $1 \leq u \leq k$ and $1 \leq k \leq n$, we denote the transition rate from a state with $q_{i_{u}}=1$ to the corresponding state with $q_{i_{u}}=0$ by $\omega_{i_{1} \ldots i_{k}}$, where $i_{u} \in\{1, \cdots, n\}$. We have $\Gamma_{i}=\{\mathbf{q}, \Delta\}(i=1, \cdots, n)$, where $q_{i}=0$, so that $\{\Delta\}=\bigcap_{i=1}^{n} \Gamma_{i}$. $\mathbf{T}$ has the following block partitioned structure:

$$
\mathbf{T}=\left[\begin{array}{cccccccccc}
d_{0} & \mathbf{B}_{1} & \mathbf{B}_{2} & \mathbf{B}_{\mathbf{3}} & \cdots & \mathbf{B}_{\mathbf{k}} & \cdots & \mathbf{B}_{1} & \cdots & \mathbf{B}_{\mathbf{n}-1} \\
& \mathbf{D}_{1} & \mathbf{C}_{12} & \mathbf{C}_{13} & \cdots & \mathbf{C}_{1 \mathbf{k}} & \cdots & \mathbf{C}_{11} & \cdots & \mathbf{C}_{1 \mathbf{n}-1} \\
& & \mathbf{D}_{\mathbf{2}} & \mathbf{C}_{23} & \cdots & \mathbf{C}_{\mathbf{2 k}} & \cdots & \mathbf{C}_{21} & \cdots & \mathbf{C}_{\mathbf{2 n}-1} \\
& & & \mathbf{D}_{3} & & & & & & \\
& & & & & \vdots & & \vdots & & \vdots \\
& & & & & \mathbf{D}_{\mathbf{k}} & \cdots & \mathbf{C}_{\mathbf{k l}} & \cdots & \mathbf{C}_{\mathbf{k n}-1} \\
& & & & & & & \mathbf{D}_{1} & \cdots & \mathbf{C}_{\mathbf{n}-1} \\
& & & & & & & & & \vdots \\
& & & & & & & & & \mathbf{C}_{\mathbf{n}-2 n-1} \\
& & & & & & & & & \mathbf{D}_{\mathbf{n}-1}
\end{array}\right]
$$


where

$d_{0}=-\left(\sum_{i=1}^{n} \omega_{i}+\sum_{i<j}^{n} \omega_{i j}+\sum_{i<j<k}^{n} \omega_{i j k}+\cdots+\omega_{123 \cdots n}\right)$

and all the unmarked entries are zeros.

The submatrices are defined as below. The dimensionality of $\mathbf{B}_{\mathbf{l}}$ is $1 \times$ $\left(\begin{array}{l}n \\ l\end{array}\right)(1 \leq l \leq n-1), \mathbf{C}_{\mathbf{k l}}$ is $\left(\begin{array}{l}n \\ k\end{array}\right) \times\left(\begin{array}{l}n \\ l\end{array}\right)(1 \leq k \leq n-2,2 \leq l \leq n-1)$ and $\mathbf{D}_{\mathbf{k}}$ is $\left(\begin{array}{l}n \\ k\end{array}\right) \times\left(\begin{array}{l}n \\ k\end{array}\right)(1 \leq k \leq n-1)$. If $\mathbf{q}=\left(q_{1}, \cdots, q_{n}\right)$ and $\mathbf{r}=\left(r_{1}, \cdots, r_{n}\right)$ are two states in $E \backslash\{\bar{\Delta}\}$, we denote by $b_{\mathbf{q r}}, c_{\mathbf{q r}}$ and $d_{\mathbf{q r}}$ the corresponding element of submatrices $\mathbf{B}_{\mathbf{l}}, \mathbf{C}_{\mathbf{k l}}$ and $\mathbf{D}_{\mathbf{k}}$, respectively. $b_{\mathbf{q r}}, c_{\mathbf{q r}}$ and $d_{\mathbf{q r}}$ will be zero unless one of the following holds:

1. $r_{i_{1}}=r_{i_{2}}=\cdots=r_{i_{u}}=0(1 \leq u \leq l)$.

Then

$b_{\mathrm{qr}}=\omega_{i_{1} i_{2} \cdots i_{l}} \quad(1 \leq l \leq n-1)$.

2. There is a $u$ such that $q_{i_{u}}=r_{i_{u}}=0(1 \leq u \leq k)$,

and $q_{j_{v}} \neq r_{j_{v}}, r_{j_{v}}=0(1 \leq v \leq l-k)$.

Then

$$
\begin{aligned}
c_{\mathbf{q r}}=\omega_{j_{1} \cdots j_{l-k}}+\sum_{u=1}^{k} \omega_{i_{u} j_{1} \cdots j_{l-k}}+\omega_{i_{1} \cdots i_{k} j_{1} \cdots j_{l-k}} & (1 \leq k \leq n-2,2 \leq l \leq n-1) .
\end{aligned}
$$

3. There is a $u$ such that $q_{i_{u}}=r_{i_{u}}=0(1 \leq u \leq k)$.

Then

$d_{\mathbf{q r}}=d_{0}+\sum_{u=1}^{k} \omega_{i_{u}}+\sum_{u<v}^{k} \omega_{i_{u} i_{v}}+\cdots+\omega_{i_{1} \cdots i_{k}} \quad(1 \leq k \leq n-1)$,

where $i_{u} \in\{1, \cdots, n\}$ and $j_{v} \in\{1, \cdots, n\}$.

To define $\beta$, let $\mathbf{Y}=\left(Y_{1}, \cdots, Y_{n}\right)$ be a random vector taking values in $\{1, \cdots, m\}$ and $p_{j_{1} \cdots j_{n}}=P\left\{Y_{1}=j_{1}, \cdots, Y_{n}=j_{n}\right\}$, where $j_{h}$ ranges over $1, \cdots, m$ for each $h=1, \cdots, n$. Thus if $\beta_{\mathbf{q}}$ is the element of $\beta$ corresponding to the state $q$, we have

$\beta_{\mathbf{q}}= \begin{cases}p_{q_{1} \cdots q_{n}} & \text { if } q_{i} \neq 0(i=1, \cdots, n), \\ 0 & \text { otherwise. }\end{cases}$

This completes the specification of the standard MPH representation of the MVE.

Consider the simplest, bivariate case, where $n=2$ and $m=3$. Then the state-space consists of the four elements $(1,1),(1,0),(0,1)$ and $(0,0)$, where $\Gamma_{1}=\{(0,1),(0,0)\}$, and $\Gamma_{2}=\{(1,0),(0,0)\}$, so that $\Delta=(0,0)$. Then $\mathbf{T}$ is $3 \times 3$ matrix 
$\mathbf{T}=\left[\begin{array}{ccc}-\left(\omega_{1}+\omega_{2}+\omega_{12}\right) & \omega_{2} & \omega_{1} \\ 0 & -\left(\omega_{1}+\omega_{12}\right) & 0 \\ 0 & 0 & -\left(\omega_{2}+\omega_{12}\right)\end{array}\right]$

The initial distribution may be written in the form $\beta=(1,0,0,0)$. From the results of Assaf et al.(1984), $\bar{F}\left(t_{1}, t_{2}\right)=P\left\{Y_{1}>t_{1}, Y_{2}>t_{2}\right\}$ has the following closed form

$$
\begin{aligned}
\bar{F}\left(t_{1}, t_{2}\right) & =\alpha e^{\mathbf{T} t_{2}} \mathbf{g}_{2} e^{\mathbf{T}\left(t_{1}-t_{2}\right)} \mathrm{g}_{1} \mathbf{e} & \text { if } & t_{1} \geq t_{2} \geq 0 \\
& =\alpha e^{\mathbf{T} t_{1}} \mathbf{g}_{1} e^{\mathbf{T}\left(t_{2}-t_{1}\right)} \mathrm{g}_{2} \mathbf{e} & \text { if } & t_{2} \geq t_{1} \geq 0
\end{aligned}
$$

where

$\alpha=(1,0,0), \quad g_{1}=\left[\begin{array}{lll}1 & 0 & 0 \\ 0 & 1 & 0 \\ 0 & 0 & 0\end{array}\right], \mathrm{g}_{2}=\left[\begin{array}{lll}1 & 0 & 0 \\ 0 & 0 & 0 \\ 0 & 0 & 1\end{array}\right]$, and $\mathbf{e}=\left[\begin{array}{l}1 \\ 1\end{array}\right]$.

They yield

$$
\begin{array}{rlrl}
\bar{F}\left(t_{1}, t_{2}\right) & =e^{-\left(\omega_{1}+\omega_{12}\right) t_{1}-\omega_{2} t_{2}} & \text { if } & t_{1} \geq t_{2} \geq 0 \\
& =e^{-\omega_{1} t_{1}-\left(\omega_{2}+\omega_{12}\right) t_{2}} \quad \text { if } & t_{2} \geq t_{1} \geq 0
\end{array}
$$

consequently we get

$\bar{F}\left(t_{1}, t_{2}\right)=e^{-\omega_{1} t_{1}-\omega_{2} t_{2}-\omega_{12} \max \left(t_{1}, t_{2}\right)}$,

which gives the bivariate exponential (BVE) distribution of Marshall and Olkin (1967).

\section{DESCRIPTION OF MODEL}

We consider a queueing system which has arbitrary $c$ servers in parallel. Assume the Poisson process $X(t)$ with rate $\alpha$ governs the occurrence of an arrival, the Poisson process $Y_{i}(t)$ with rate $\beta_{i}$ governs the occurrence of service completion to server $i$ for $1 \leq i \leq c$, and the Poisson process $Z(t)$ with rate $\gamma$ governs the occurrence of an arrival and service completion to all $c$ servers simultaneously. Assume all the Poisson processes are independent. Let $T$ be the interarrival time of a customer, $S_{i}$ be the service time of server $i$ for $1 \leq i \leq c$. Then the joint distribution of the interarrival time $T$ and $c$ service times $S_{i}(1 \leq i \leq c)$ is given precisely as follows(see, for example, Barlow and Proschan (1981)):

$$
\begin{aligned}
P\left\{T>t, S_{1}>s_{1}, S_{2}\right. & \left.>s_{2}, \cdots, S_{c}>s_{c}\right\} \\
& =\exp \left[-\alpha t-\sum_{i=1}^{c} \beta_{i} s_{i}-\gamma \max \left(t, s_{1}, s_{2}, \cdots, s_{c}\right)\right] .
\end{aligned}
$$

This multivariate exponential distribution was first studied by Marshall and 
Olkin (1967). It is well known that $T$ has a marginal exponential distribution with rate $\lambda=\alpha+\gamma, S_{i}(1 \leq i \leq c)$ has a marginal exponential distribution with parameter $\mu_{i}=\beta_{i}+\gamma(1 \leq i \leq c)$. We remark that it is usually assumed in the literature $\gamma>0$ to have a physical interpretation of the component lifetime (see Barlow and Proschan (1981)). In the case $\gamma>0$, the interarrival time of a customer and the service time of server $i(1 \leq i \leq c)$ can be expressed, respectively, as follows:

$T=\min (U, W), \quad S_{i}=\min \left(V_{i}, W\right), \quad 1 \leq i \leq c$,

where $U, V_{i}(1 \leq i \leq c)$ and $W$ are independent exponential random variables, with parameters (rates) $\alpha, \beta_{i}(1 \leq i \leq c)$ and $\gamma$, respectively. As the same manner as the ordinary $\mathrm{M} / \mathrm{M} / \mathrm{c}$ queueing system, if we let $U$ be an interarrival time and $V_{i}$ be service time of server $i$ for $1 \leq i \leq c$, then any channel finishes service independently on an arrival. However, by introducing an independent exponential random variable $W$ in (16), in addition to that, all $c$ channels accomplish service simultaneously when a customer arrives. We need the condition that $\alpha, \beta_{i}(1 \leq i \leq c)$ and $\gamma$ are nonnegative and $\alpha, \beta_{i}>-\gamma($ for the arrival and service rates to be positive). To ensure that the queueing system is stable, we also need $\lambda<\sum_{i=1}^{c} \mu_{i}$, or $\alpha<\sum_{i=1}^{c} \beta_{i}+(c-1) \gamma$. Furthermore, since $\lambda>0$, we have $\sum_{i=1}^{c} \beta_{i}+(c-1) \gamma>\alpha>-\gamma$.

\section{THE STEADY STATE PROBABILITY VECTOR}

The model under consideration can be studied as a continuous time Markov chain with state-space $B \cup M . B$ is the set of all $c$-tuples $\mathbf{b}=\left(b_{1}, \cdots, b_{c}\right)$ with $b_{i} \in\{0,1\}(1 \leq i \leq c)$ and $\sum_{i=1}^{c} b_{i}=k(0 \leq k \leq c-1)$. The number of states in the boundary state is $\sum_{i=0}^{c-1}\left(\begin{array}{l}c \\ i\end{array}\right) \cdot b_{i}=1$ means that the channel is occupied by a customer, whereas $b_{i}=0$ means that it is not occupied. To put it concretely, all boundary states are

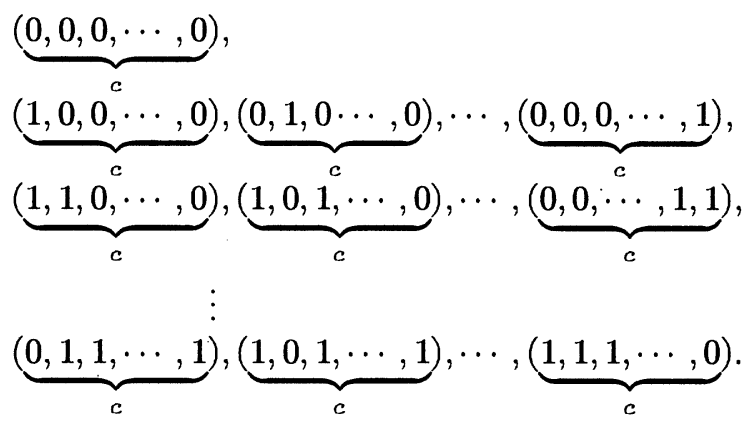


On the other side, $M$ is the set of $\left(n ; m_{1}, \cdots, m_{c}\right)(n \geq c)$ with $m_{i} \in$ $\{0,1\}(1 \leq i \leq c)$ and $\sum_{i=1}^{c} m_{i}=k(k=1, c)$, where $n$ denotes the number of customers in the system. The number of these states is $\sum_{i=0}^{1}\left(\begin{array}{l}c \\ i\end{array}\right) . m_{i}=1$ means that the customer in channel $i$ is one who just joined the service facility lastly of all customers on service. We will say that he is a new customer. $m_{i}=0$ means that the customer in channel $i$ is not a new customer. We will say that he is an old customer. In the concrete, all $c$-tuples $\left(m_{1}, \cdots, m_{c}\right)$ are

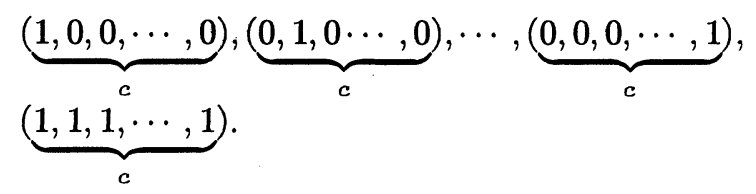

It is assumed that when a simultaneous completion of service occurs, a customer must enter into the channel with the lower number, and a simultaneous completion of service cannot occur when either of channels is empty since it is not realistic. If we look upon served customers including an arrived customer as new customers when there are still empty channels except the occupied channel, whereas we look upon only an arrived customer as a new customer when there are no empty channels except the occupied channel, then $b_{i}=1$ means that the customer is new. By ordering the states as described above, the infinitesimal generator of the continuous time Markov chain has the following block partitioned structure:

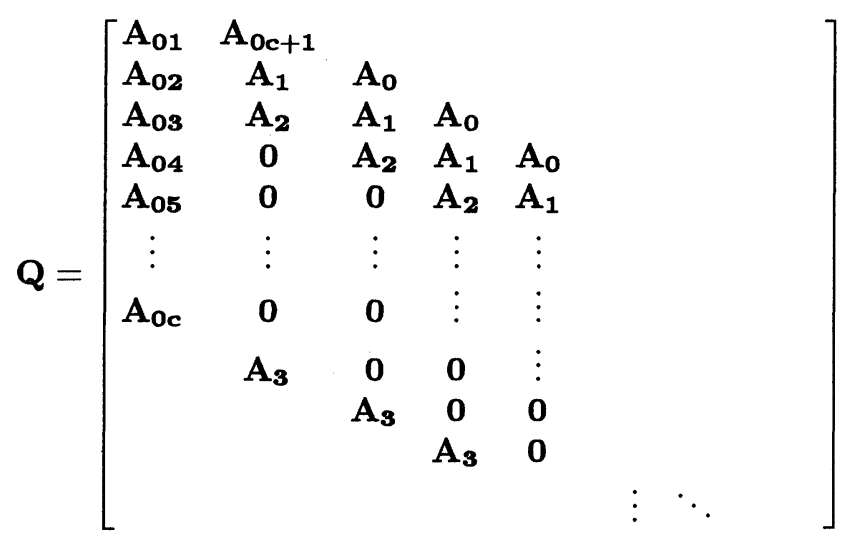

where all the unmarked entries are zeros. The submatrices are defined as below. The dimensionality of $\mathbf{A}_{01}$ is $\left(\sum_{i=0}^{c-1}\left(\begin{array}{l}c \\ i\end{array}\right)\right) \times\left(\sum_{i=0}^{c-1}\left(\begin{array}{l}c \\ i\end{array}\right)\right), \mathbf{A}_{0 \mathbf{j}}(2 \leq j \leq c)$ are $\left(\sum_{i=0}^{1}\left(\begin{array}{l}c \\ i\end{array}\right)\right) \times\left(\sum_{i=0}^{c-1}\left(\begin{array}{l}c \\ i\end{array}\right)\right), \mathbf{A}_{0 c+1}$ is $\left(\sum_{i=0}^{c-1}\left(\begin{array}{l}c \\ i\end{array}\right)\right) \times\left(\sum_{i=0}^{1}\left(\begin{array}{l}c \\ i\end{array}\right)\right), \mathbf{A}_{\mathbf{0}}, \mathbf{A}_{1}, \mathbf{A}_{\mathbf{2}}, \mathbf{A}_{\mathbf{3}}$ are 
$\left(\sum_{i=0}^{1}\left(\begin{array}{l}c \\ i\end{array}\right)\right) \times\left(\sum_{i=0}^{1}\left(\begin{array}{l}c \\ i\end{array}\right)\right) .0$ is a $\left(\sum_{i=0}^{1}\left(\begin{array}{l}c \\ i\end{array}\right)\right) \times\left(\sum_{i=0}^{1}\left(\begin{array}{l}c \\ i\end{array}\right)\right)$ zero matrix. More specifically, $\mathbf{S}=\left[\begin{array}{ccccccc}\mathbf{0} & & & & & & \\ \mathbf{E}_{\mathbf{c - 1}} \mathbf{e} & \mathbf{D}_{\mathbf{c}-1}^{\mathbf{T}} & & & & & \\ \mathbf{E}_{\mathbf{c - 2}} \mathbf{e} & \mathbf{C}_{\mathbf{c - 2 , c - 1}}^{\mathbf{T}} & \mathbf{\mathbf { D } _ { \mathbf { c } - 2 } \mathbf { T }} & & & & \\ \vdots & \vdots & \vdots & \mathbf{D}_{1}^{\mathbf{T}} & & & \\ \mathbf{E}_{\mathbf{k}} \mathbf{e} & \mathbf{C}_{\mathbf{k}, \mathbf{c}-1}^{\mathbf{T}} & \cdots & \mathbf{C}_{\mathbf{k}, \mathbf{1}}^{\mathbf{T}} & \mathbf{D}_{\mathbf{k}}^{\mathbf{T}} & & \\ \vdots & \vdots & \cdots & \vdots & \vdots & & \\ \mathbf{E}_{\mathbf{2}} \mathbf{e} & \mathbf{C}_{\mathbf{2}, \mathbf{c}-1}^{\mathbf{T}} & \cdots & \mathbf{C}_{\mathbf{2}, 1}^{\mathbf{T}} & \cdots & \mathbf{D}_{\mathbf{2}}^{\mathbf{T}} & \\ \mathbf{E}_{\mathbf{1}} \mathbf{e} & \mathbf{C}_{1, \mathbf{c}-1}^{\mathbf{T}} & \cdots & \mathbf{C}_{1,1}^{\mathbf{T}} & \cdots & \cdots & \mathbf{D}_{1}^{\mathbf{T}}\end{array}\right]$

where $\mathbf{G}^{\mathbf{T}}$ is a matrix whose elements are reversed fully, that is,

$\mathbf{G}=\left[\begin{array}{lll}g_{11} & g_{12} & g_{13} \\ g_{21} & g_{22} & g_{23} \\ g_{31} & g_{32} & g_{33}\end{array}\right], \quad \mathbf{G}^{\mathbf{T}}=\left[\begin{array}{lll}g_{33} & g_{32} & g_{31} \\ g_{23} & g_{22} & g_{21} \\ g_{13} & g_{12} & g_{11}\end{array}\right]$

and the transpose of $\mathrm{e}$ is $(1,1, \cdots, 1)$ with a proper size.

$\mathbf{E}_{\mathbf{k}}=-\left(\mathbf{D}_{\mathbf{k}}^{\mathbf{T}}+\sum_{l=k+1}^{c-1} \mathbf{C}_{\mathbf{k} \mathbf{l}}^{\mathbf{T}}\right)(1 \leq k \leq c-1)$ and $\mathbf{B}_{\mathbf{l}}, \mathbf{C}_{\mathbf{k l}}$ and $\mathbf{D}_{\mathbf{k}}$ are given by $(7),(8)$ and (9) respectively, where

$\omega_{i}=\beta_{i}, \quad 1 \leq i \leq c$

$\omega_{i j}=\omega_{i j k}=\cdots=\omega_{123 \cdots c}=0$.

Furthermore, if $\mathbf{v}=\left(v_{1}, \cdots, v_{c}\right)$ and $\mathbf{w}=\left(w_{1}, \cdots, w_{c}\right)$ are two states, we denote by $\mathbf{H}=\left(h_{\mathbf{v w}}\right)$ which is an upper triangular matrix. $h_{\mathbf{v w}}=\lambda$ if one of the following holds, otherwise $h_{\mathrm{vw}}=0$, where $\eta$ denotes the number of occupied channels:

1. $\eta=0$.

Then

$\mathbf{w}=\mathbf{v}_{\mathbf{0}} \cup\left\{v_{\min \left(i_{\nu}\right)}=1\right\}=\mathbf{v}_{\mathbf{0}} \cup\left\{v_{1}=1\right\}$,

where $v_{0}=(0, \cdots, 0)$ (i.e. an empty state) and $i_{\nu} \in\{1, \cdots, c\}$.

$2.1 \leq \eta \leq c-2$.

Then

$\begin{array}{lll}\mathbf{w}=\mathbf{v} \cup\left\{v_{\min \left(i_{\nu}\right)}=1\right\} & \text { for } & v_{1}=1 \\ \mathbf{w}=\mathbf{v} \cup\left\{v_{1}=1\right\} & \text { for } & v_{1}=0\end{array}$

Consequently, 
$\mathbf{A}_{\mathbf{0 1}}=\mathbf{S}-\lambda \mathbf{I}+\mathbf{H}$

As the same manner, we can obtain $\mathbf{A}_{\mathbf{0 c}+\mathbf{1}}$ for $\eta=c-1$. If $\mathbf{v}$ and $\mathbf{q}=(c ; \mathbf{w})$ are two states, we denote by $\mathbf{A}_{\mathbf{0 c}+1}=\left(h_{\mathbf{v q}}\right)$. Then $h_{\mathbf{v q}}=\lambda$ if the following holds, otherwise $h_{\mathrm{vq}}=0$ :

$\mathbf{w}=\mathbf{v}_{\mathbf{0}} \cup\left\{v_{i_{\nu}}=1\right\} \quad$ for $\quad v_{i_{\nu}}=0$.

On the other hand, denoting by

$\mathbf{A}_{\mathbf{0 2}}=\mathbf{e}\left(h_{\mathbf{w}}\right)$,

$h_{\mathbf{w}}=\gamma$ when $\mathbf{w}=(1,0, \cdots, 0)$ and $h_{\mathbf{w}}=\beta_{\nu}$ if the following holds, otherwise $h_{\mathbf{w}}=0$ :

$\mathbf{w}=\mathbf{w}_{1} \cup\left\{w_{i_{\nu}}=0\right\}, \quad 1 \leq \nu \leq c$,

where $\mathrm{w}_{1}=(1,1, \cdots, 1)$.

Similarly, denoting by $\mathbf{A}_{\mathbf{2}}=\mathbf{e}\left(h_{\mathbf{w}}\right), h_{\mathbf{w}}=\beta_{\nu}$ if the following holds, otherwise $h_{\mathbf{w}}=0$ :

$\mathbf{w}=\mathbf{w}_{0} \cup\left\{w_{i_{\nu}}=1\right\}, \quad 1 \leq \nu \leq c$,

where $\mathbf{w}_{\mathbf{0}}=(0,0, \cdots, 0)$.

We also denote by $\mathbf{A}_{\mathbf{0} \mathbf{j}}=\mathbf{e}\left(h_{\mathbf{w}}\right)(3 \leq j \leq c)$. Then $h_{\mathbf{w}}=\gamma$ if the following holds, otherwise $h_{\mathbf{w}}=0$ :

$\mathbf{w}=(\underbrace{1, \cdots, 1}_{j-1}, 0, \cdots, 0)$.

And

$\mathbf{A}_{\mathbf{0}}=\alpha \mathbf{I}$,

$\mathbf{A}_{1}=-\left(\alpha+\sum_{i=1}^{c} \beta_{i}+\gamma\right) \mathbf{I}$

$\mathbf{A}_{\mathbf{3}}=\mathbf{e}(\underbrace{0,0, \cdots, 0}_{c}, \gamma)$.

Let $\mathbf{x}$ be the vector of steady state probabilities associated with $\mathbf{Q}$, such that

$\mathbf{x} \mathbf{Q}=\mathbf{0} \quad$ and $\quad \mathbf{x e}=\mathbf{1}$.

Let us partition $\mathbf{x}$ as $\mathbf{x}=\left(\mathbf{x}_{\mathbf{b}}, \mathbf{x}_{\mathbf{c}}, \mathbf{x}_{\mathbf{c}+1}, \cdots\right)$ where $\mathbf{x}_{\mathbf{b}}$ is a $1 \times\left(\sum_{i=0}^{c-1}\left(\begin{array}{l}c \\ i\end{array}\right)\right)$ vector associated with the boundary state $\mathbf{b}$ and $\mathbf{x}_{\mathbf{i}}(i \geq c)$ are $1 \times\left(\sum_{i=0}^{1}\left(\begin{array}{l}c \\ i\end{array}\right)\right)$ vectors 
associated with the states m. Following Neuts (1981),

$\mathbf{x}_{\mathbf{i}}=\mathbf{x}_{\mathbf{c}} \mathbf{R}^{\mathbf{i}-\mathbf{c}}, \quad i \geq c+\mathbf{1}$,

where the rate matrix $\mathbf{R}$ is of order $\left(\sum_{i=0}^{1}\left(\begin{array}{l}c \\ i\end{array}\right)\right)$ and the minimal nonnegative solution to the matrix nonlinear equation

$\mathbf{A}_{0}+\mathbf{R} \mathbf{A}_{1}+\mathbf{R}^{\mathbf{2}} \mathbf{A}_{2}+\mathbf{R}^{\mathbf{c}} \mathbf{A}_{3}=\mathbf{0}$.

The boundary condition is

$\mathbf{x}_{\mathbf{b}} \mathbf{A}_{\mathbf{0 1}}+\sum_{i=1}^{c-1} \mathbf{x}_{\mathbf{i}+\mathbf{c}-\mathbf{1}} \mathbf{A}_{\mathbf{0 i}+\mathbf{1}}=\mathbf{0}$

$x_{b} A_{0 c+1}+x_{c} A_{1}+x_{c+1} A_{2}+x_{2 c-1} A_{3}=0$.

The normalizing condition is

$\mathbf{x}_{\mathrm{b}} \mathbf{e}+\mathbf{x}_{\mathrm{c}}(\mathbf{I}-\mathbf{R})^{-1} \mathbf{e}=\mathbf{1}$.

Let $\mathbf{p}$ be the steady state probabilities, assuming that the queue is never empty, which has elements $p(j)\left(1 \leq j \leq \sum_{i=0}^{1}\left(\begin{array}{l}c \\ i\end{array}\right)\right)$. We can determine $\mathbf{p}$, by solving the system

$\mathbf{p A}=\mathbf{0} \quad$ and $\quad \mathbf{p e}=1$,

where the conservative matrix is given by

$\mathbf{A}=\mathbf{A}_{\mathbf{0}}+\mathbf{A}_{1}+\mathbf{A}_{2}+\mathbf{A}_{\mathbf{3}}$.

Following Neuts (1981), the stability condition is

$\mathbf{p} \mathbf{A}_{\mathbf{0}} \mathbf{e}<\mathbf{p}\left[\mathbf{A}_{\mathbf{2}}+(c-1) \mathbf{A}_{\mathbf{3}}\right] \mathbf{e}$.

By solving the system (36) and substituting $p(j)$ into (38), the stability condition becomes

$\alpha<\sum_{i=1}^{c} \beta_{i}+(c-1) \gamma$,

which agrees with the result obtained in the previous section.

Substituting (37) into (33), we have

$(\mathbf{I}-\mathbf{R})\left[-\gamma \sum_{i=2}^{c-1} \mathbf{R}^{\mathbf{i}}-\left(\sum_{i=1}^{c} \beta_{i}+\gamma\right) \mathbf{R}+\alpha \mathbf{I}\right] \mathbf{e}=\mathbf{0}$.

Since $\mathbf{I}-\mathbf{R}$ is nonsingular, we get

$\left[-\gamma \sum_{i=2}^{c-1} \mathbf{R}^{\mathbf{i}}-\left(\sum_{i=1}^{c} \beta_{i}+\gamma\right) \mathbf{R}+\alpha \mathbf{I}\right] \mathbf{e}=\mathbf{0}$. 
In particular, for $c=3$,

$\mathbf{R}=\zeta^{-1} \mathbf{I}$

is the minimal solution of the equation (41), where $\zeta$ is

$\zeta=\left\{\sum_{i=1}^{3} \beta_{i}+\gamma+\left[\left(\sum_{i=1}^{3} \beta_{i}+\gamma\right)^{2}+4 \alpha \gamma\right]^{\frac{1}{2}}\right\} /(2 \alpha)$.

If the stability condition (39) is satisfied, $\zeta^{-1}<1$. Denoting the steady state probability vector by $\mathbf{x}_{\mathbf{i}}=\left(x_{i 1}, x_{i 2}, x_{i 3}, x_{i 4}\right)(i \geq 3)$, the steady state probabilities $x_{i}$, where $i$ represents the number of customers in the system, are given by

$x_{i}=\sum_{j=1}^{4} x_{i j}, \quad i \geq 4$.

Substituting (42) into (32) and (44), we obtain

$$
\begin{array}{ll}
\mathbf{x}_{\mathbf{i}}=\mathbf{x}_{\mathbf{3}} \zeta^{3-i} \mathbf{I}, & i \geq 4, \\
x_{i j}=x_{3 j} \zeta^{3-i}, & i \geq 4,1 \leq j \leq 4, \\
x_{i}=x_{3} \zeta^{3-i}, & i \geq 4 .
\end{array}
$$

If $\gamma=0$, an interarrival and three service times are mutually independent, hence by letting $\gamma \rightarrow 0$, the steady state probabilities $x_{i}(i \geq 4)$ in (47) coincide with those of the ordinary $\mathrm{M} / \mathrm{M} / 3$ queueing system.

\section{EFFECT OF THE CORRELATION PARAMETER}

In this section, we study the effect of the correlation parameter on some random variable, with the marginal distribution of interarrival and service times fixed. When $\lambda$ and $\sum_{i=1}^{c} \beta_{i}+\gamma=\kappa$ are fixed, varying the correlation parameter $\gamma$, we investigate the effect of $\gamma$ on the subtraction of an interarrival time from the minimum of $c$ service times.

A random variable $X$ is said to be less than or equal to $Y$ in increasing convex ordering if $E f(X) \leq E f(Y)$ for all function $f$ which is increasing and convex, denoted $X \leq_{i c} Y$. A necessary and sufficient condition for $X \leq_{i c} Y$ is

$\int_{y}^{\infty} P\{X>t\} d t \leq \int_{y}^{\infty} P\{Y>t\} d t \quad$ for all $y$.

For more details about increasing convex ordering and other stochastic orderings, the readers are referred to Ross (1983) and Stoyan (1983).

Define

$S=\min \left(S_{1}, S_{2}, \cdots, S_{\mathrm{c}}\right)$,

$\Delta=S-T$, 
where $S_{1}, S_{2}, \cdots, S_{c}$ and $T$ are random variables defined in (16). We obtain the following same lemma as Chao's one (1995) because the MVE is a distribution with exponential minimums.

Lemma. $\Delta$ is decreasing in $\gamma$ in increasing convex ordering.

That is, $\Delta\left(\gamma_{1}\right) \geq_{i c} \Delta\left(\gamma_{2}\right)$ if $\gamma_{1} \leq \gamma_{2}$.

Proof. Using the results given by Barlow and Proschan (1981),

$P\{S>s \mid T=t\}= \begin{cases}e^{-(\kappa-\gamma) t}, & t>s, \\ \frac{\alpha}{\alpha+\gamma} e^{-\gamma(s-t)-(\kappa-\gamma) s}, & t \leq s .\end{cases}$

Hence, by conditioning on $T$ and using (51), we obtain if $x>0$,

$$
\begin{aligned}
P\{\Delta>x\} & =\int_{0}^{\infty} P\{S-T>x \mid T=t\}(\alpha+\gamma) e^{-(\alpha+\gamma) t} d t \\
& =\alpha e^{-\kappa x} \int_{0}^{\infty} e^{-(\alpha+\kappa) t} d t \\
& =\frac{\alpha}{\alpha+\kappa} e^{-\kappa x} .
\end{aligned}
$$

If $x \leq 0$, then

$$
\begin{aligned}
P\{\Delta>x\} & =\int_{0}^{\infty} P\{S-T>x \mid T=t\}(\alpha+\gamma) e^{-(\alpha+\gamma) t} d t \\
& =\int_{0}^{-x} P\{S-T>x \mid T=t\}(\alpha+\gamma) e^{-(\alpha+\gamma) t} d t \\
& +\int_{-x}^{\infty} P\{S-T>x \mid T=t\}(\alpha+\gamma) e^{-(\alpha+\gamma) t} d t \\
& =\int_{0}^{-x}(\alpha+\gamma) e^{-(\alpha+\gamma) t} d t+(\alpha+\gamma) e^{-(\kappa-\gamma) x} \int_{-x}^{\infty} e^{-(\alpha+\kappa) t} d t \\
& =1-\frac{\kappa-\gamma}{\alpha+\kappa} e^{(\alpha+\gamma) x} .
\end{aligned}
$$

To prove $\Delta$ is decreasing in $\gamma$ in increasing convex ordering, it suffices to show that $\int_{y}^{\infty} P\{\Delta>x\} d x$ is decreasing in $\gamma$ for all $y$. To this end, we again consider two cases. First, $y \geq 0$. This case is trivial since from (52),

$P\{\Delta>x\}=\frac{\alpha}{\alpha+\kappa} e^{-\kappa x}=\left(1-\frac{\kappa}{\lambda+\kappa-\gamma}\right) e^{-\kappa x}$.

This is obviously decreasing in $\gamma$, hence so $\int_{0}^{\infty} P\{\Delta>x\} d x$. If $y \leq 0$, then by (52) and (53),

$$
\begin{aligned}
\int_{y}^{\infty} P\{\Delta>x\} d x & =\int_{y}^{0} P\{\Delta>x\} d x+\int_{0}^{\infty} P\{\Delta>x\} d x \\
& =\int_{y}^{0}\left[1-\frac{\kappa-\gamma}{\alpha+\kappa} e^{(\alpha+\gamma) x}\right] d x+\int_{0}^{\infty} \frac{\alpha}{\alpha+\kappa} e^{-\kappa x} d x \\
& =-y-\frac{\kappa-\gamma}{(\alpha+\gamma)(\alpha+\kappa)}\left[1-e^{(\alpha+\gamma) y}\right]+\frac{\kappa}{\alpha+\kappa} \\
& =-y-\frac{1}{\lambda}+\frac{1}{\kappa}+\frac{1}{\lambda} e^{\lambda y}-\frac{1}{\lambda+\kappa-\gamma} e^{\lambda y},
\end{aligned}
$$

therefore it is also decreasing in $\gamma$.

To summarize, we have shown that for any $y, \int_{y}^{\infty} P\{\Delta>x\} d x$ is a decreasing function of $\gamma$. This proves that $\Delta$ is decreasing in $\gamma$ in increasing convex ordering.Q.E.D. 
Our multiserver model is different from a single server model treated by Chao (1995) though both models introduce the multivariate exponential distribution of Marshall and Olkin. Even if we change our model, for example, for letting $T_{n}$ be the interarrival time between customer $n$ and customer $n+1$ and $S_{n}=\min \left(S_{1}, S_{2}, \cdots, S_{c}\right)$ be $c$ service times ahead of customer $n$, we cannot derive the same theorem as one that Chao (1995) derived since a multiserver model carries complicated behavior.

\section{CONCLUDING REMARKS}

For an $\mathrm{M} / \mathrm{M} / \mathrm{c}$ queueing system with an interarrival time and $c$ service times having the multivariate exponential distribution of Marshall and Olkin, we have calculated the steady state probability vector using its MPH representation by a matrix-geometric approach, and defined dependency between an interarrival time and $c$ service times as the rate of the simultaneous occurrence of an arrival and service completion of all channels, and showed that the subtraction of an interarrival time from the minimum of $c$ service times is monotonically decreasing in the dependency in increasing convex ordering. It allowed us to treat our multiserver model that the multivariate exponential distribution of Marshall and Olkin is a distribution with exponential minimums. We found out the class of systems that enjoy the monotonic dependency effect on some random variable, so we could say that our model will contribute to the development of practical queueing models with interdependent arrival and service processes.

\section{REFERENCES}

Assaf, D., Langberg, N.A., Savits, T.H. and Shaked, M. (1984) Multivariate phase-type distributions. Operations Research, 32, 688-702.

Barlow, R.E. and Proschan, F. (1981) Statistical theory of reliability and life testing probability models, To begin with. Silver Spring, MD.

Chao, X. (1995) Monotone effect of dependency between interarrival and service times in a simple queueing system. Operations Research Letters, 17, 47-51.

Conolly, B.W. and Choo, Q.H. (1979) The waiting time process for a generalized correlated queue with exponential demand and service. SIAM Journal on Applied Mathematics, 37, 236-275.

Esary, J.D. and Marshall, A.W. (1974) Multivariate distributions with exponential minimums. The Annals of Statistics, 2, 84-98.

Hadidi, N. (1981) Queues with partial correlation. SIAM Journal on Applied Mathematics, 40, 467-475.

Hadidi, N. (1981) Further result on queues with partial correlation. Operations Research, 33, 203-209.

Langaris, C. (1987) Busy period analysis of a correlated queue with exponen- 
tial demand and service. Journal of Applied Probability, 24, 476-485.

Marshall, A.W. and Olkin, I. (1967) A multivariate exponential distribution. Journal of the American Statistical Association, 62, 30-44.

Neuts, M.F. (1981) Matrix-geometric solutions in stochastic models: An algorithmic approach. The Johns Hopkins University Press, Baltimore.

Nishida, T., Watanabe, R. and Tahara, A. (1974) Poisson queue with correlated two servers. Technology Reports of the Osaka University, 24, 403409.

Nishida, T. and Yoneyama, K. (1977) Correlated multiserver queue with random input. Mathematica Japonica, 22, 395-401.

O'Cinneide, C.A. and Raftery, A.E. (1989) A continuous multivariate exponential distribution that is multivariate phase type. Statistics and Probability Letters, 7, 323-325.

Raftery, A.E. (1984) A continuous multivariate exponential distribution. Communications in Statistics -Theory and Methods, 13, 947-965.

Ross, S. (1983) Stochastic processes. Wiley, New York.

Stoyan, D. (1983) Comparison methods for queues and other stochastic models. Wiley, New York.

Yoneyama, K. (1996) The waiting time distribution of correlated multiserver queue. Mathematica Japonica, 43, 569-576.

Yoneyama, K., Miyamura, T. and Ishii, H. (1997) A matrix-geometric solution for correlated multiserver queues. Proceedings of Symposium on Performance Models for Information Communication Networks, 394-402.

Yoneyama, K., Miyamura, T. and Ishii, H. (1997) M/M/2 queue with interdependent arrival and service processes. Technology Reports of the Osaka University, 47, 151-159.

\section{BIOGRAPHY}

K. Yoneyama is Associate Professor at the Department of Management and Informatics, Himeji College of Hyogo. He received B.E., M.E. and Dr. of Engineering from Osaka University. His current research is in correlated queueing systems. He has over 20 published papers in various journals which include Computers and Mathematics with Applications.

T. Miyamura has completed his Bachelor of Engineering from Osaka University. Currently he is doing research for his Master of Engineering in the field of Operations Research at Osaka University.

H. Ishii is Professor at the Faculty of Engineering, Osaka University. He received B.E., M.E. and Dr. of Engineering from Kyoto University. His papers have appeared in European Journal of Operational Research, Operations Research, Mathematical Programming, Annals of Operations Research, Discrete Applied Mathematics, Networks, Journal of the Operations Research Society of Japan and other journals. 